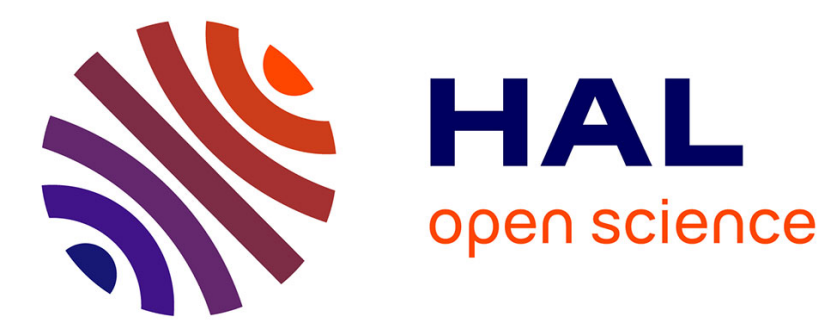

\title{
X-ray diagnosis of the pressure Induced Mott Nonmetal-Metal Transition
}

\author{
A. Levy, F. Dorchies, A. Benuzzi-Mounaix, A. Ravasio, F. Festa, V. Recoules, \\ O. Peyrusse, N. Amadou, E. Brambrink, T. Hall, et al.
}

\section{- To cite this version:}

A. Levy, F. Dorchies, A. Benuzzi-Mounaix, A. Ravasio, F. Festa, et al.. X-ray diagnosis of the pressure Induced Mott Nonmetal-Metal Transition. Physical Review Letters, 2012, 108 (5), pp.055002. 10.1103/PhysRevLett.108.055002 . hal-01561849

\section{HAL Id: hal-01561849 \\ https://hal.science/hal-01561849}

Submitted on 13 Jan 2022

HAL is a multi-disciplinary open access archive for the deposit and dissemination of scientific research documents, whether they are published or not. The documents may come from teaching and research institutions in France or abroad, or from public or private research centers.
L'archive ouverte pluridisciplinaire HAL, est destinée au dépôt et à la diffusion de documents scientifiques de niveau recherche, publiés ou non, émanant des établissements d'enseignement et de recherche français ou étrangers, des laboratoires publics ou privés. 


\title{
X-Ray Diagnosis of the Pressure Induced Mott Nonmetal-Metal Transition
}

\author{
A. Lévy, ${ }^{1, *}$ F. Dorchies, ${ }^{2}$ A. Benuzzi-Mounaix,${ }^{1}$ A. Ravasio, ${ }^{1}$ F. Festa, ${ }^{1,3}$ V. Recoules,${ }^{3}$ O. Peyrusse,${ }^{2}$ N. Amadou, ${ }^{1}$ \\ E. Brambrink, ${ }^{1}$ T. Hall, ${ }^{4}$ M. Koenig, ${ }^{1}$ and S. Mazevet ${ }^{3,5}$ \\ ${ }^{1}$ LULI, École Polytechnique, CNRS, CEA, UPMC, route de Saclay, 91128 Palaiseau, France \\ ${ }^{2}$ Université de Bordeaux-CNRS-CEA, Centre Lasers Intenses et Applications (CELIA), Talence, F-33405, France \\ ${ }^{3}$ CEA, DAM, DIF, F-91297 Arpajon, France \\ ${ }^{4}$ Physics Department, University of Essex-Colchester, United Kingdom \\ ${ }^{5}$ LUTH, Observatoire Paris Meudon, CNRS, Université Paris Diderot, 5 place Janssen, 92195 Meudon Cedex, France
}

(Received 17 June 2011; published 30 January 2012)

\begin{abstract}
The evolution of the $K$-edge $\mathrm{x}$-ray absorption near-edge spectroscopy (XANES) spectrum is investigated for an aluminum plasma expanding from the solid density down to $0.5 \mathrm{~g} / \mathrm{cm}^{3}$, with temperatures lying from 5 down to $2 \mathrm{eV}$. The dense plasma is generated by nanosecond laser-induced shock compression. These conditions correspond to the density-temperature region where a metal-nonmetal transition occurs as the density decreases. This transition is directly observed in XANES spectra measurements through the progressive formation of a preedge structure for densities around $1.6 \mathrm{~g} / \mathrm{cm}^{3}$. Ab initio calculations based on density functional theory and a jellium model have been efficiently tested through direct comparison with the experimental measurements and show that this preedge corresponds to the relocalization of the $3 p$ atomic orbital as the system evolves from a dense plasma toward a partially ionized atomic fluid.
\end{abstract}

DOI: 10.1103/PhysRevLett.108.055002

The nonmetal-metal transition observed during the compression or expansion of a dense plasma has been the object of a large amount of theoretical and experimental studies in the past decades [1-7]. In this context, the experimental work was based on the electrical conductivity or optical reflectivity measurements to characterize this complex regime that lies between a dense plasma (with electronic structure close that of a heated solid) and a partially ionized fluid where perturbation theories employed in atomic and low density plasma physics can be used. The renewed interest in the accurate description of this thermodynamic regime, called warm dense matter, lies in its implication for a growing number of applications ranging from inertial confinement fusion capsules to the description of planetary inner cores [8].

For metallic systems such as aluminum, the Mott transition is driven by pressure [9]. When the pressure decreases, the interatomic distances increase, and the metal turns to an insulator as the individual atomic wave functions cease to overlap. This process is reversible since a pressure increase induces the metallization of the material. This phenomenon is rather generic and is found for many elements. To predict the density-temperature region where this happens is a stringent test for theoretical modeling that historically struggled at describing this effect also referred to as "pressure ionization" in atomic and dense plasma physics. In taking account of pressure ionization, it is also particularly important to calculate plasma transport properties such as absorption or opacity. At a given density, it limits the contribution from some of the atomic (ionic) states of the isolated atom or ion [10].
PACS numbers: 52.50.Lp, 32.30.Rj, 52.25.Os, 71.30.th

For dense plasmas, the extreme temperature $(T)$ and density $(\rho)$ conditions at which this phenomenon occurs have long prevented accurate measurements. Indeed, it requires both to bring matter close to solid density with high temperature (few eVs) and simultaneously to characterize its transport properties. This regime has been recently reached for several atomic or molecular systems and for plasma states produced by using either gas gun techniques [1], exploding wires [2,3], or high power lasers $[4,11]$. Up to now, this transition has just been indirectly observed through conductivity or optical reflectivity measurements. In this Letter, we present a direct signature of the electronic structure evolution in the metal-nonmetal transition using time-resolved $\mathrm{x}$-ray absorption near-edge spectroscopy (XANES) measurement during the unloading of a laser-shocked aluminum foil. The results are compared to two different theoretical models based on ab initio calculations [12,13] and a jellium model [14] as a result of the simultaneous measurement of $(\rho, T)$ conditions during the $\mathrm{x}$-ray probing. This allows one to determine at which density this transition occurs.

The experiment, schematized in Fig. 1, has been performed on the LULI2000 laser facility at the Laboratoire pour l'Utilisation des Lasers Intenses (LULI). It consisted of generating a shock compressed $\mathrm{Al}$ sample by using a $150 \mathrm{~J}, 500 \mathrm{ps}$ frequency-doubled Nd-glass laser pulse (drive beam) operating at a central wavelength of $527 \mathrm{~nm}$. This pulse launches a shock wave into a solid multilayer foil structured as follows: $15 \mu \mathrm{m}$ plastic $(\mathrm{CH}$ ablator) $/ 1 \mu \mathrm{m} \mathrm{Al}$ (studied sample) $/ 5 \mu \mathrm{m}$ (CH-tamped layer) as illustrated in Fig. 1. The thermodynamic characteristics of the sample were determined by using rearside 


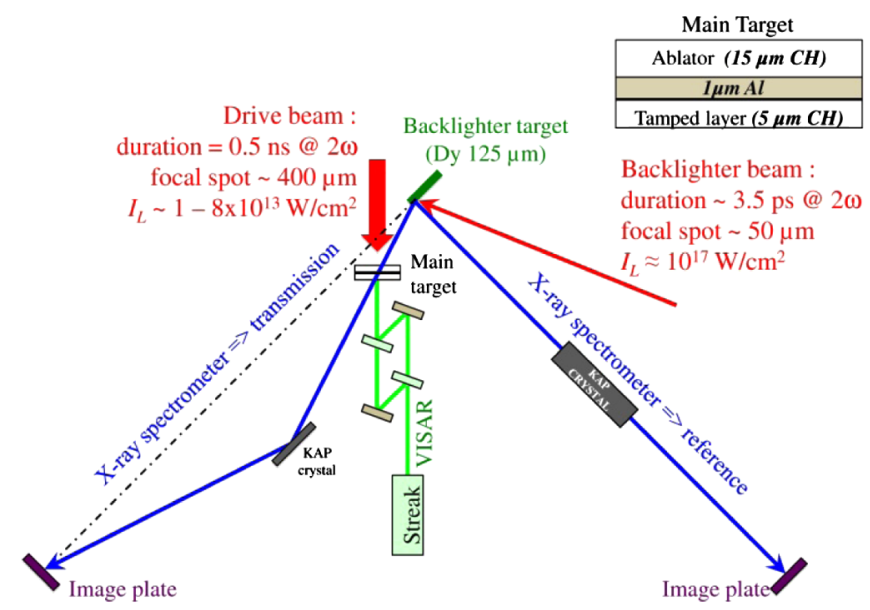

FIG. 1 (color online). Scheme of the experimental setup.

optical diagnostics: velocity interferometer system for any reflector [15] (VISAR) and self-optical pyrometry (SOP). Both, coupled with a streak camera, allow the determination of the shock mean velocity by the measurement of the transit through the $\mathrm{CH}$ layer. VISAR gives also the instantaneous shock velocity by the detection of fringe displacement induced by the shock wave propagation in the rearside plastic layer. The shock velocities were $>30 \mathrm{~km} \mathrm{~s}^{-1}$ corresponding to pressure above $\sim 6.6$ Mbar. 1D hydrodynamic simulations (MULTI [16]) have been performed to reproduce the rearside optical measurements and retrieve the density and temperature temporal evolutions in the Al layer. These simulations have been performed by using the SESAME 3717 equation of states. During compression, the Al sample is strongly shocked to twice the solid density $\left(2 \rho_{\text {solid }}\right)$ and a temperature of about $5 \mathrm{eV}$. When the shock wave reaches the target rearside, the plastic expands into the vacuum and an unloading wave travels back into the target. When this wave reaches the Al layer, its density decreases under $\rho_{\text {solid }}$.

To probe the change in the electronic structure of the sample under these thermodynamic conditions, we used a XANES diagnostic coupled with an ultrafast $\mathrm{x}$-ray source. This source, optimized in a previous experiment [17], takes advantage of $M$-band emission from high $Z$ plasmas and is expected to last less than 10 ps. This isotropic emission was obtained from the interaction of the picosecond beam of the LULI2000 facility (20 J at central wavelength $=$ $527 \mathrm{~nm}, 3.5$ ps-backlighter beam) with a dysprosium target. The absorption spectra are retrieved around the $\mathrm{Al} K$ edge $(1.5596 \mathrm{keV})$ thanks to two independent spectrometers coupled to image plates (following the principle described in Ref. [18]) and, respectively, measuring the direct emission from the $\mathrm{x}$-ray source and the transmitted signal through the sample. Positioning the $\mathrm{x}$-ray detector out of the focal plane of the conical potassium acid phthalate crystals, we were able to image the sample with a $35 \mu \mathrm{m}$ spatial resolution (perpendicular to the spectral dispersion). The $\mathrm{x}$-ray source covered the whole shocked area corresponding to $400 \mu \mathrm{m}$ diameter in the sample plane. In order to ensure a good transverse homogeneity, we selected in the sample image the central $315 \mu \mathrm{m}$ wide region. Finally, using hydrodynamic simulations, we calculated the delay needed between the two laser pulses to probe the $\mathrm{Al}$ foil during its expansion from solid density down to $0.5 \mathrm{~g} / \mathrm{cm}^{3}$.

Using the experimental setup described above, we obtained different $\mathrm{x}$-ray absorption spectra, each corresponding to a single shot in a precise region of the phase diagram $(\rho, T)$. The hydrodynamic conditions deduced from the VISAR and SOP measurements are summarized in the legend of Fig. 2(a). The delay between the two pulses was varied from $800 \mathrm{ps}$ up to $2.5 \mathrm{~ns}$. This leads to densities varying from 2.1 down to $0.5 \mathrm{~g} / \mathrm{cm}^{3}$ and temperatures from 5 down to $2 \mathrm{eV}$. The error bars in densities and temperatures take into account the uncertainty in the shock velocity measurement, the relative delay between the drive and backlighter beams, and the use of different equation of state SESAME tables and results in relative errors of $\Delta T / T \sim \pm 0.22$ and $\Delta \rho / \rho \sim \pm 0.08$. These tables have been largely validated experimentally by different works [19] and especially in this low density regime by Ref. [7]. Moreover, according to these hydrodynamic simulations, the temperature and density profiles are almost uniform along the sample thickness.

The x-ray spectra are reported in Fig. 2(a). They are compared with calculated spectra for the same $(\rho, T)$ couples by using two different models [Figs. 2(b) and 2(c)]. All these curves are normalized far above the $K$ edge (at a photon energy of about $1.6 \mathrm{keV}$ where no modification of the spectrum is expected). We further point out that the experiment measures the transmission of the x-ray beam through an expanding $\mathrm{Al}$ foil but fixed areal density. As such, we measure the "linear absorption" that is the photoabsorption cross section times the density at which the measurement is performed.

Figure 2(a) shows that XANES spectra are drastically modified during the plasma expansion. At highest densities and temperatures, the spectrum is typical of a warm dense aluminum plasma, in agreement with previous results reported by using proton heating. The disappearance of any characteristic structures in the spectrum is related to the loss of short range order at such temperatures [20,21]. As both the temperature and density decrease, we first see a steepening of the edge and, for the lowest density-temperature conditions, the continuous formation of a sharp preedge. We define as "preedge" the structure emerging from the solid density aluminum plasma XANES spectrum. We performed $a b$ initio electronic structure calculations and used a jellium model to characterize this effect.

The spectra reported in Fig. 2(b) are obtained by using the $a b$ initio approach described in detail in Ref. [21]. The method consists in performing quantum molecular 

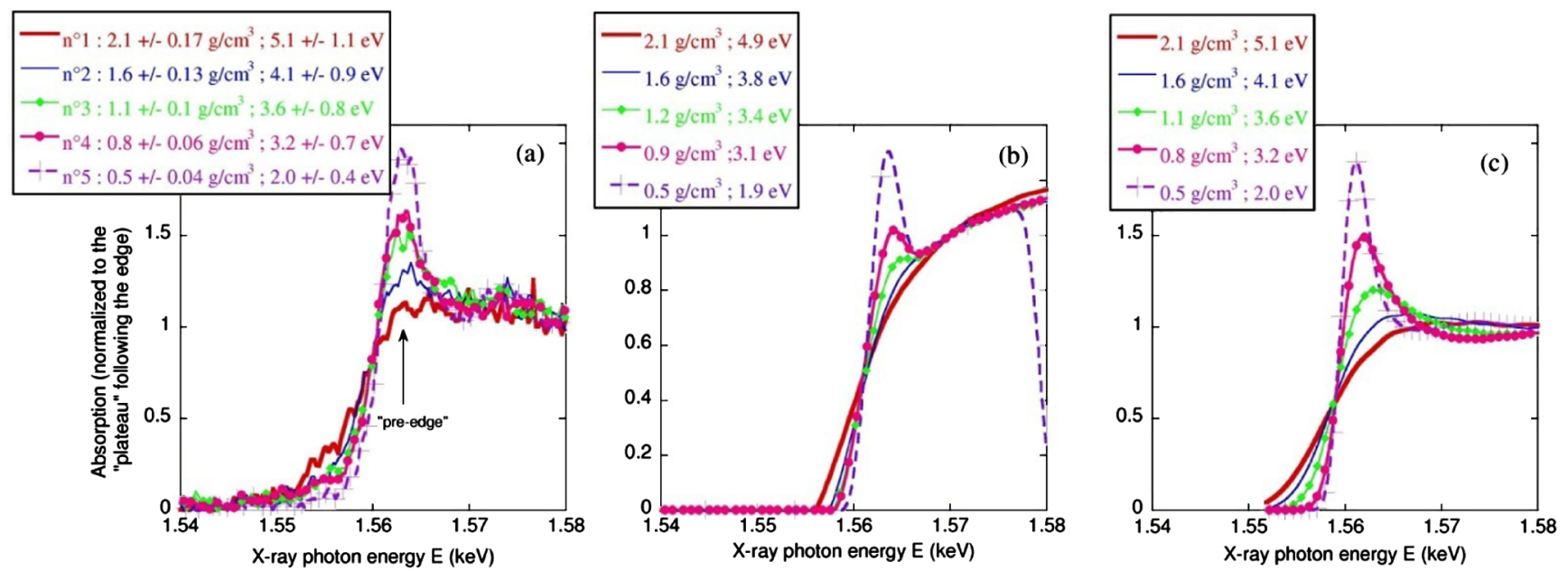

FIG. 2 (color online). X-ray absorption spectra around the $\mathrm{Al} K$ edge for different $(\rho, T)$ conditions: (a) Experimental measurements [the $(\rho, T)$ conditions have been evaluated by using hydrodynamic simulations and, respectively, correspond to the pump-probe delays of $0.8,1,1.25,1.5$, and $2.5 \mathrm{~ns}$ ]. (b) Ab initio calculations. (c) Average atom model in a jellium calculation.

dynamic calculations to simulate the aluminum sample at a given density or temperature condition. We used ABINIT electronic code [22] and generated a projected augmented wave (PAW) pseudopotential $[23,24]$ within the framework of the Perdew-Burke-Ernzerhof parametrization of density functional theory [25] using the ATOMPAW generator [26]. The PAW pseudopotential calculated consists in three outer electrons $\left(3 s^{2} 3 p^{1}\right)$ and a cutoff radius $r_{c}=1.7 a_{0}$, where $a_{0}$ is the Bohr radius. Its validity was previously established against all electron and experimental data for the $\mathrm{Al}$ cold curve [21]. Moreover, comparing ab initio pressures and the SESAME ones, we found an excellent agreement, completing the consistency of the procedure and enhancing the confidence in this PAW pseudopotential. We performed the electronic structure calculation by using a plane cutoff $15 \mathrm{Ha}$, which provides convergence in the total energy better than $10^{-6} \mathrm{Ha}$. To obtain the optical response in the $\mathrm{x}$-ray domain, we applied the linear response theory as expressed within the Kubo-Greenwood formulation [12] on selected snapshots over the trajectory and by using a $2 \times 2 \times 2 k$-point grid as defined within the MonkhorstPack scheme [26].

The spectra reported in Fig. 2(c) are obtained by using the well-known average atom model in a jellium (or electron gas model) [14], i.e., an average atom embedded into a uniform electron density $\rho_{e 0}$ neutralized by a positive background simulating the ionic charges. Therefore, the problem is reduced to the response of the electronic density when a positive charge $Z$ is immersed into a jellium. After convergence, the corresponding potential is used for calculating the continuum orbitals (with $l=1$ ) as well as the $1 s$ bound orbital that are involved in the calculation of the photoionization cross section for various energies around the edge. In these calculations, the exchange potential comes from the generalized gradient approximation formulation of Perdew and Wang [27].
Figure 2 shows that both theoretical predictions are in quantitative agreement with the experimental results. As the density and temperature decrease, the preedge progressively appears for densities around $1 \mathrm{~g} / \mathrm{cm}^{3}$ as previously predicted in different studies [5,7]. However, for the first time, the experimental results obtained during this campaign are sufficiently precise to test the limits of theories, as this feature is clearly noticeable at $1.6 \mathrm{~g} / \mathrm{cm}^{3}$ on the experimental spectra while both $a b$ initio and average atom calculations suggest its appearance at lower densities $\left(\sim 1.1 \mathrm{~g} / \mathrm{cm}^{3}\right)$. This is consistent with the underestimation of the band gap energy characteristic of density-functional theory methods. Indeed, as the system expands, one expects that smaller band gaps would lead to relocalization at lower densities. In the same vein, calculations performed with the average atom model but with a simple local-density approximation functional that predicts a larger gap gives the relocalization at a lower density than $1.1 \mathrm{~g} / \mathrm{cm}^{3}$.

Earlier [7] $a b$ initio calculations of the $\mathrm{Al}$ index of refraction and optical conductivity showed a transition occurring at densities $\leq 0.1 \mathrm{~g} / \mathrm{cm}^{3}$. This phenomenon was associated to the formation of the $3 s-3 p$ bound states. At the higher densities and temperatures probed here, we are investigating the relocalization of the $3 p$ state preceding the formation of the bound atomic states. This is illustrated by the variation of the density of states (DOS) shown in Fig. 3. At the highest density, the DOS is uniform and characteristic of a simple metal (i.e., of a free electron gas). As the density decreases, two sharp maxima appear, the $3 s-3 p$ band, typical of relocalization. At even lower densities, this will turn into atomic bound states. As XANES is directly proportional to the $p$ component of the DOS, it is direct evidence of the $3 p$ band formation. This is corroborated by the simpler average atom in jellium calculations. In this model the preedge turns out to be a density-induced $3 p$ continuum resonance [28]. 


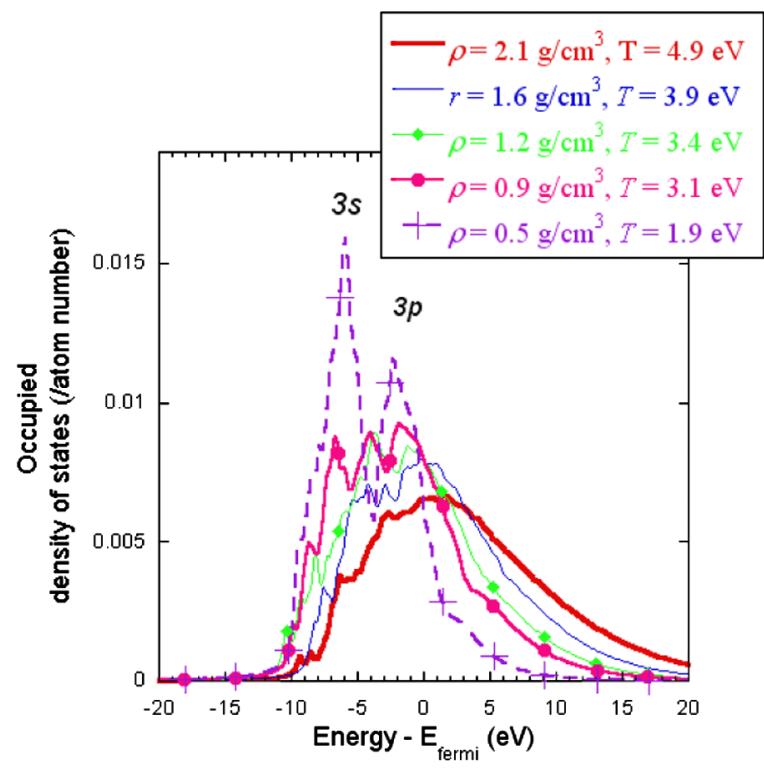

FIG. 3 (color online). Ab initio occupied density of states for the experimental $(\rho, T)$ conditions.

Finally, we should point out that the transition evidenced in the present study is related to the relocalization of the electronic orbitals of the $\mathrm{Al}^{+}$ion and not the neutral atom as the sample temperature was in all cases sufficiently high (above $2 \mathrm{eV}$ ). Ab initio calculations showed previously that the effective ionization fraction varies from 1 to 0.1 when the temperature decreases from 2.6 to $0.9 \mathrm{eV}$ [7]. In addition, we remark that $1 \mathrm{~g} / \mathrm{cm}^{3}$ corresponds to the density at which transition occurs between the thermally activated conductivity and pressure induced metallic state (overlapping orbital's). The measurements performed in this study are clear evidence that this also corresponds to the relocalization of the $3 p$ state that manifests itself as a preedge in the XANES spectrum.

In summary, we performed XANES measurement near the $K$ edge during the expansion of a laser shock compressed dense aluminum plasma. We observed the formation of a preedge at the lowest densities, around $1.6 \mathrm{~g} / \mathrm{cm}^{3}$, that was identified as the relocalization of a $3 p$-like bound state of $\mathrm{Al}^{+}$ions as predicted by ab initio molecular dynamics simulations and calculations performed by using an average atom model. The detailed behavior of the XANES spectrum as it evolves as a function of pressure and density remains, however, to be improved, as substantial differences are noticeable between the theoretical predictions and the experimental measurements. Combined with the equation of state and conductivity measurements, the results presented in the current study clearly show that $K$-edge XANES measurements provide a stringent test for theoretical modeling and unprecedented details on the evolution of the electronic structure of matter under extreme density and temperature conditions.

We acknowledge useful discussion with P. Renaudin as well as the support of the GENCI program for providing computational time under the program GEN6046.

*anna.levy@polytechnique.edu

[1] S. T. Weir, A.C. Mitchell, and W. J. Nellis, Phys. Rev. Lett. 76, 1860 (1996).

[2] A. W. De Silva and J. D. Katsouros, Phys. Rev. E 57, 5945 (1998); A. W. De Silva and G. B. Vunni, Phys. Rev. E 83, 037402 (2011).

[3] J.F. Benage, W. R. Shanahan, and M.S. Murillo, Phys. Rev. Lett. 83, 2953 (1999).

[4] G. W. Collins et al., Science 281, 1178 (1998).

[5] M. P. Desjarlais, J. D. Kress, and L. A. Collins, Phys. Rev. E 66, 025401 (2002).

[6] V. Recoules et al., Phys. Rev. E 66, 056412 (2002).

[7] S. Mazevet et al., Phys. Rev. E 71, 016409 (2005).

[8] I. Baraffe et al., Rep. Prog. Phys. 73, 016901 (2010).

[9] N. F. Mott, Rev. Mod. Phys. 40, 677 (1968).

[10] D. G. Hummer et al., Astrophys. J. 331, 794 (1988).

[11] P. K. Patel et al., Phys. Rev. Lett. 91, 125004 (2003).

[12] V. Recoules and S. Mazevet, Phys. Rev. B 80, 064110 (2009).

[13] S. Mazevet et al., AIP Conf. Proc. 730, 139 (2004).

[14] O. Peyrusse, J. Quant. Spectrosc. Radiat. Transfer 99, 469 (2006), and references therein.

[15] L. M. Barker et al., J. Appl. Phys. 43, 4669 (1972).

[16] R. Ramis et al., Comput. Phys. Commun. 49, 475 (1988).

[17] M. Harmand et al., Phys. Plasmas 16, 063301 (2009).

[18] A. Lévy et al., Rev. Sci. Instrum. 81, 063107 (2010).

[19] C. E. Ragan III, Phys. Rev. A 25, 3360 (1982); 29, 1391 (1984); A. C. Mitchell and W. J. Nellis, J. Appl. Phys. 52, 3363 (1981); R. F. Trunin et al., JETP 81, 464 (1995); R. F. Trunin et al., Sov. Phys. JETP 68, 356 (1972).

[20] A. Mancic et al., Phys. Rev. Lett. 104, 035002 (2010).

[21] S. Mazevet et al., High Energy Density Phys. 6, 84 (2010); S. Mazevet and G. Zerah, Phys. Rev. Lett. 101, 155001 (2008).

[22] X. Gonze et al., Comput. Mater. Sci. 25, 478 (2002); X. Gonze et al., Comput. Phys. Commun. 180, 2582 (2009).

[23] P. E. Blöchl, Phys. Rev. B 41, 5414 (1990).

[24] J. P. Perdew, K. Burke, and M. Ernzerhof, Phys. Rev. Lett. 77, 3865 (1996).

[25] M. Torrent et al., Comput. Mater. Sci. 42, 337 (2008).

[26] N. A. W. Holzwarth et al., Comput. Phys. Commun. 135, 329 (2001).

[27] J. P. Perdew and Y. Wang, Phys. Rev. B 33, 8800 (1986).

[28] R. M. More, Adv. At. Mol. Phys. 21, 305 (1985). 